\title{
Independent roles of Fgfr2 and Frs2 $\alpha$ in ureteric epithelium
}

\author{
Sunder Sims-Lucas ${ }^{1}$, Brian Cusack ${ }^{1}$, Veraragavan P. Eswarakumar ${ }^{2}$, Jue Zhang ${ }^{3}$, Fen Wang ${ }^{3}$ and \\ Carlton M. Bates ${ }^{1,4, *}$
}

\begin{abstract}
SUMMARY
Mice with conditional deletion of fibroblast growth factor receptor 2 ( $F g f r 2)$ in the ureteric bud using a Hoxb7cre line (Fgfr2 ${ }^{U B-/-}$ ) develop severe ureteric branching defects; however, ureteric deletion of fibroblast growth factor receptor substrate $2 \alpha(F r s 2 \alpha)$, a key docking protein that transmits fibroblast growth factor receptor intracellular signaling (Frs $2 \alpha^{U B-l-}$ ) leads to mild ureteric defects. Mice with point mutations in the Frs $2 \alpha$ binding site of Fgfr2 ( $F g f r 2^{L R / L R}$ ) have normal kidneys. The aim of this study was to determine the relationship between Fgfr2 and Frs $2 \alpha$ in the ureteric lineage. Mice with ureteric deletion of both Fgfr2 and Frs $2 \alpha$ (Fgfr2/Frs $2 \alpha^{U B-/}$ ) were compared with Frs $2 \alpha^{U B-I-}$ and Fgfr2 ${ }^{U B-I-}$ mice. To avoid potential rescue of Fgfr1 forming heterodimers with Fgfr $2^{L R}$ alleles to recruit Frs $2 \alpha$, compound mutant mice were generated with ureteric deletion of Fgfr1 and with Fgfr $2^{L R / L R}$ point mutations ( $\left.F g f r 1^{U B-I-} F g f r 2^{L R / L R}\right)$. At E13.5, three-dimensional reconstructions and histological assessment showed that, whereas Fgfr $2^{U B-1-}$ kidneys had more severe ureteric branching defects than Frs $2 \alpha^{U B-1-}$, Fgfr2 ${ }^{U B-1-}$ kidneys were indistinguishable from Fgfr2/Frs $2 \alpha^{U B-1-}$. At later stages, however, Fgfr2/Frs $2 \alpha^{U B-1-}$ kidneys were more severely affected than either Fgfr2 ${ }^{U B-I-}$ or Frs $2 \alpha^{U B-I-}$ kidneys. Taken together, although Fgfr2 and Frs $2 \alpha$ have crucial roles in the ureteric lineage, they appear to act separately and additively.
\end{abstract}

KEY WORDS: Fibroblast growth factor receptor 2, Frs2 $\alpha$, Ureteric epithelium, Mouse

\section{INTRODUCTION}

Metanephric kidney development occurs via interactions of the ureteric bud (UB) and metanephric mesenchyme (MM) (Costantini and Kopan, 2010). The UB grows out from the Wolffian duct, receiving signals from the adjacent MM. The UB then elongates, contacts the MM and then branches within the MM over several generations. Terminal UB tips induce surrounding MM-derived nephron progenitors to condense and then differentiate into epithelial nephrons. Cortical stromal mesenchyme also forms and ultimately differentiates into interstitial cells.

Fibroblast growth factor receptor (Fgfr) signaling is crucial for kidney development. Fgfrs are receptor tyrosine kinases (RTKs) with four signaling family members and 22 known mammalian ligands (Powers et al., 2000). Many investigators have shown that Fgf ligands and receptors have crucial roles in renal mesenchymal and ureteric lineages, often with conditional knockout approaches (Barasch et al., 1997; Barasch et al., 1999; Celli et al., 1998; Hains et al., 2008; Li et al., 2006; Nguyen et al., 1996; Ohuchi et al., 2000; Perantoni et al., 1995; Poladia et al., 2006; Qiao et al., 2001; Qiao et al., 1999; Revest et al., 2001; Zhao et al., 2004). We demonstrated that conditional deletion of Fgfr2 using a Hoxb7cre line (targeting the UB) results in aberrant ureteric and cortical stromal patterning (Zhao et al., 2004).

\footnotetext{
${ }^{1}$ Rangos Research Center, Children's Hospital of Pittsburgh of UPMC, Pittsburgh, PA 15201, USA. ${ }^{2}$ Department of Orthopaedics and Rehabilitation, Yale University School of Medicine, New Haven, CT 06520, USA. ${ }^{3}$ Center for Cancer Biology and Nutrition, Institute of Biosciences and Technology, Texas A\&M, Houston, TX 77030 USA. ${ }^{4}$ Division of Nephrology, Department of Pediatrics, University of Pittsburgh School of Medicine, Pittsburgh, PA 15201, USA.

*Author for correspondence (batescm@upmc.edu)
}

Accepted 14 January 2011
Fgfr signaling is mediated via docking adapter proteins that transmit intracellular signaling. One well-characterized adapter is fibroblast growth factor receptor substrate $2 \alpha$ (Frs $2 \alpha$; Frs 2 - Mouse Genome Informatics) (Gotoh, 2008). Frs $2 \alpha$ binds constitutively in the receptor juxtamembrane region and upon Fgfr simulation becomes phosphorylated, ultimately activating Erk, Akt and protein kinase $\mathrm{C}$ (PKC) $\lambda$ and $\xi$. Frs $2 \alpha$ also serves as an adapter protein for other RTKs such as neurotrophin receptors and Ret (Gotoh, 2008). Global deletion of Frs $2 \alpha$ results in early embryonic lethality, presumably due to the inability of some RTKs to signal properly (Hadari et al., 2001).

Given that Frs $2 \alpha$ is utilized by Fgfr 2 and Ret, both of which are crucial for ureteric morphogenesis (Schuchardt et al., 1994; Zhao et al., 2004), we investigated the role of Frs $2 \alpha$ in the UB by conditional deletion with our Hoxb7cre line (Sims-Lucas et al., 2009 b). Inactivation of Frs $2 \alpha$ in ureteric tissues resulted in mild renal hypoplasia with slightly fewer ureteric tips and normal stromal patterning. Furthermore, mice with point mutations in Fgfr2 that replace amino acids Leu-424 (L) and amino acid Arg$426(\mathrm{R})$ with Ala residues ( $\left.F g f r 2^{L R / L R}\right)$ to prevent Frs $2 \alpha$ binding to the receptor had normal kidneys (Eswarakumar et al., 2006; SimsLucas et al., 2009b).

To understand the relationship between Fgfr2 and Frs $2 \alpha$ in the $\mathrm{UB}$, we examined mice with combined conditional deletion of Fgfr 2 and Frs $2 \alpha$ with our Hox $7 b c r e$ line $\left(F g f r 2 / F r s 2 \alpha^{U B-/-}\right)$ in comparison with deletion of Frs $2 \alpha$ alone $\left(F r s 2 \alpha^{U B-/-}\right)$ or Fgfr 2 alone $\left(F g f r 2^{U B-/-}\right)$. To avoid potential rescue of intact Fgfr1 forming heterodimers with $\mathrm{Fgfr} 2^{\mathrm{LR}}$ point mutants to recruit $\mathrm{Frs} 2 \alpha$, we generated mice with conditional deletion of $F g f r l$ from the UB and with the $F g f r 2^{L R / L R}$ point mutations ( $\left.F g f r 1^{U B-/} F g f r 2^{L R / L R}\right)$. At E13.5, three-dimensional (3D) reconstructions and histological assessment confirmed that $F g f r 2^{U B-/-}$ kidneys had more severe ureteric branching defects than Frs $2 \alpha^{U B-/}$, but that $F g f r 2^{U B-/-}$ kidneys were indistinguishable from $F g f r 2 / F r s 2 \alpha^{U B-/}$. At 
later stages, however, Fgfr $2 / F r s 2 \alpha^{U B-/-}$ kidneys were more severely affected than $F g f r 2^{U B-/-}$ or $F r s 2 \alpha^{U B-/-}$ kidneys. Fgfr $1^{U B-/-} F g f r 2^{L R / L R}$ mice had phenotypically normal kidneys. Thus, although Fgfr2 and Frs $2 \alpha$ each have crucial roles in the ureteric lineage, they appear to act independently and additively.

\section{MATERIALS AND METHODS}

\section{Mouse strains}

Our Hoxb7cre line has been shown to drive efficient deletion of Fgfrl, Fgfr2 and Frs $2 \alpha$ in the UB (Sims-Lucas et al., 2009b; Zhao et al., 2004). For this study, $\mathrm{Fgfr} 2^{\text {Lox/Lox }}$ [gift from Dr David Ornitz, Washington University, St Louis, MO, USA (Yu et al., 2003)] were bred with Frs $2 \alpha^{\text {Lox/Lox }}$ mice (Lin et al., 2007) to produce Fgfr $2^{\text {Lox/Lox }} F r s 2 \alpha^{\text {Lox/Lox }}$ mice. These mice were bred with $H o x b 7 \mathrm{creEGFP} \mathrm{Tg}^{\mathrm{Tg}+}$ to generate Hoxb 7 creEGFP ${ }^{T g /+} \mathrm{Fg} f \mathrm{r}^{\mathrm{Lox} /+} \mathrm{Frs} 2 \mathrm{\alpha}^{\mathrm{Lox} /+}$ mice. These mice were bred back with $F g f r 2^{\text {Lox/Lox }} F r s 2 \alpha^{L o x / L o x}$ to produce mice with ureteric epithelial deletion of $F r s 2 \alpha \quad\left(F r s 2 \alpha^{U B-/-}\right), \quad F g f r 2 \quad\left(F g f r 2^{U B-1-}\right)$ or both (Fgfr $2 / F r s 2 \alpha^{U B-/-}$ ) (see Table $\mathrm{S} 1$ in the supplementary material). We used similar breeding strategies to generate mice with ureteric deletion of $\mathrm{Fg} f \mathrm{rl}$ ( $F g f r 1^{U B-1-}$ ), with point mutations in the Frs $2 \alpha$ binding site on Fgfr2 $\left(F g f r 2^{L R / L R}\right)$, or both $\left(F g f r 1^{U B-1} F g f r 2^{L R / L R}\right)$ (see Table S2 in the supplementary material). When a vaginal plug was identified, noon of that day was deemed embryonic day 0.5 (E0.5).

\section{Genotyping}

Briefly, genomic DNA was isolated from tail clippings and/or embryonic tissues. Polymerase chain reaction (PCR) amplification identified the various alleles. For the HoxCreEgfp cassette, the forward primer 5'-AGCGCGATCACATGGTCCTG-3' and reverse primer 5'ACGATCCTGAGACTTCCACACT-3' yielded a 230 base pair (bp) band identifying cre. For the Frs $2 \alpha$ allele, the forward primer 5'GAGTGTGCTGTGATTGGAAGGCAG-3' and reverse primer $5^{\prime}$ GGCACGAGTGTCTGCAGACACATG-3' yielded a 224 bp wild type and a $319 \mathrm{bp}$ floxed band. For the $F g f r 2$ allele, the forward primer 5'-GTCAATTCTAAGCCACTGTCTGCC-3' and reverse primer $5^{\prime}$ CTCCACTGATTACATCTAAAGAGC-3' yielded a 307 bp wild type and a 373 bp floxed band. For the $F g f r 2^{L R}$ allele, the forward primer 5'-GAGTACCATGCTGACTGCATGC-3' and the reverse primer $5^{\prime}$ GGAGAGGCATCTCTGTTTCAAGACC- $3^{\prime}$ yielded a 225 bp wild type and a 315 bp mutant band.

\section{Histology}

Embryos and/or kidneys were fixed in 4\% paraformaldehyde (PFA) in phosphate buffered saline overnight at $4^{\circ} \mathrm{C}$. Kidneys were examined under direct light and photographed with a fluorescence microscope to visualize the ureteric tree. Other tissues were placed in $70 \%$ ethanol at $4{ }^{\circ} \mathrm{C}$ (for section histology) or dehydrated into $100 \%$ methanol at $4^{\circ} \mathrm{C}$ (for wholemount histology). Tissues used for section histology were processed, embedded into paraffin and sectioned at $7 \mu \mathrm{m}$. Sections were stained with Hematoxylin and Eosin (H\&E) or were subjected to non-radioactive in situ hybridization (ISH) for Foxd1 as described (Zhao et al., 2004).

E17.5 kidneys were also subjected to whole-mount immunostaining with antibodies against monoclonal mouse anti-calbindin- $\mathrm{D}_{28 \mathrm{k}}$ antisera (C9848, Sigma-Aldrich, MO, USA). Briefly, kidneys were rehydrated through methanol into PBST (PBS with $0.1 \%$ Tween 20 ) and blocked in $10 \%$ donkey serum. Tissues were incubated with primary antisera $(1: 100)$ overnight at $4^{\circ} \mathrm{C}$. Kidneys were washed in PBST and incubated with Alexa Fluor 488 goat anti-mouse (1:100; A11001, Invitrogen, Carlsbad, CA, USA) overnight at $4^{\circ} \mathrm{C}$. Kidneys were washed, mounted and imaged with an LSM 710 confocal microscope (Carl Zeiss MicroImaging, Thornwood, NY, USA).

E17.5 kidney sections were also subjected to aquaporin 2 (Aqp2) immunostaining. Control and $F g$ fr $2 F r s 2 a^{U B-/-}$ ( $n=3$ per genotype) paraffin kidney sections $(4 \mu \mathrm{m})$ through renal papilla were de-waxed and hydrated. After permeabilizing with $0.1 \%$ Tween 20-PBS, tissues were blocked in $5 \%$ goat serum for 30 minutes. Sections were then incubated with polyclonal rabbit anti-Aqp2 (1:100; Calbiochem, CA, USA) for 45 minutes at $37^{\circ} \mathrm{C}$. Kidneys were washed with PBS and then streptavidin-peroxidase staining was performed using an $\mathrm{ABC}$ kit (Vector Labs, CA, USA).

\section{Glomerular profiling}

P30 control, Frs $2 a^{U B-/-}, F g f r 2^{U B-/-}$ and $F g f r 2 F r s 2 a^{U B-/-}$ kidneys ( $n=3$ per genotype) were bisected in the transverse plane, fixed in 4\% PFA, processed to paraffin, and H\&E stained. From the midline plane, three 4 $\mu \mathrm{m}$ sections were obtained at $200 \mu \mathrm{m}$ intervals and every glomerulus per level was traced using Stereoinvestigator [Microbrightfield (MBF), VT, USA] to measure total and mean cross-sectional area and perimeter $(n=452$ for controls; $n=388$ for Frs $2 a^{U B-/} ; n=335$ for Fgfr $2^{U B---} ; n=153$ for Fgfr $\left.2 \mathrm{Frs}_{2 \mathrm{a}}{ }^{U B-1-}\right)$.

\section{Morphometric measurements}

E17.5 mutant and control embryos were sacrificed and weighed. Kidneys were removed, weighed and photographed. Cross-sectional area (area), and kidney long axis were measured using Image $\mathrm{J}$ analysis software (version $1.32 \mathrm{j}$ from Wayne Rasband, National Institutes of Health, USA).

\section{$3 D$ rendering}

3D reconstructions of E13.5 kidney capsules and ureteric trees were performed as described with slight modifications for skeletonizations (Sims-Lucas et al., 2009a). Briefly, image layers were generated from serial $\mathrm{H} \& \mathrm{E}$ stained sections by tracing capsules and ureteric tissues using Stereoinvestigator. Traced layers were aligned, rendering 3D images to show any traced structures. Using Neurolucida Explorer (MBF), volume and surface area were calculated for kidneys and the UB. From this, relative volumes and surface areas of ureteric epithelium (ureteric volume per kidney volume and ureteric surface area per kidney area) were determined. To skeletonize the ureteric tree, individual layers were exported into Microsoft Paint (Redmond, WA, USA) and tracings were filled in and saved as tiff files. Images were imported into Imaris software (Bitplane, Zurich, Switzerland) and a 3D skeleton of the ureteric tree was generated. Number of branch points and tips, lengths of branches (ureteric segments between two branch points), tips (ureteric segments from the last branch point to the outer cortex) and total ureteric segments (all segments including branches and tips) were calculated. Movies and still images of intact and skeletonized ureteric trees were generated.

\section{Statistical analysis}

Statistical analyses were performed using Student's $t$-test or one-way analysis of variance (ANOVA) followed by Student-Newman-Keuls posthoc tests. Values are represented as mean \pm standard deviation.

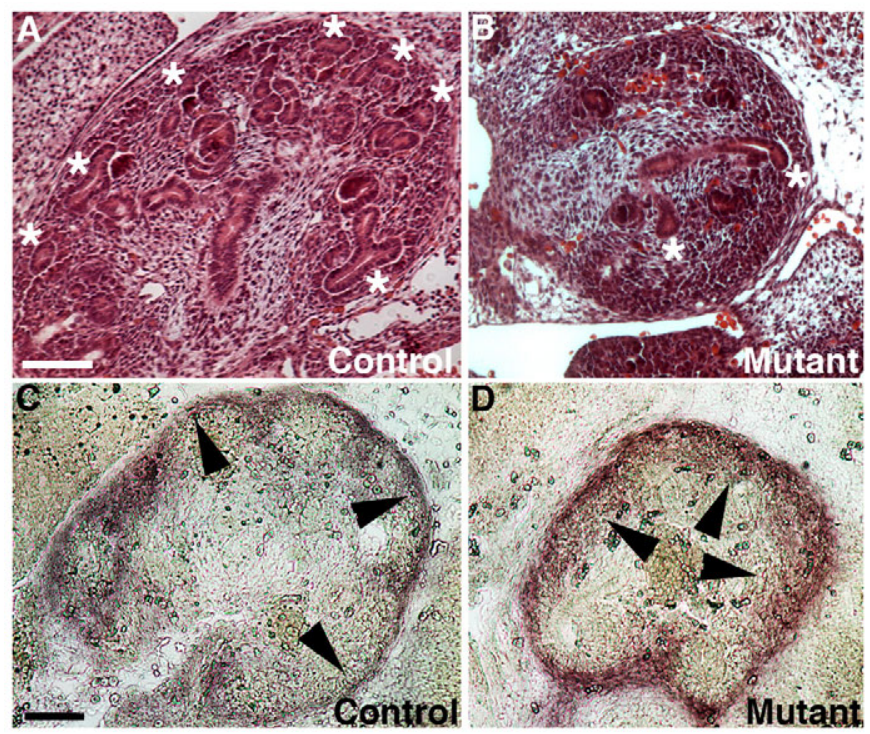

Fig. 1. Histological abnormalities in E13.5 Fgfr2/Frs2 $\alpha^{\mathrm{UB}-/-}$ kidneys. (A,B) H\&E sections show that compared with controls (A), mutants (B) have fewer ureteric tips (asterisks) and developing nephrons. (C,D) Foxd1 in situ hybridization shows that compared with cortical stroma in controls (C, arrowheads), mutants (D) have regions of aberrantly thickened cortical stroma (arrowheads). Scale bars: $100 \mu \mathrm{m}$. 


\section{RESULTS AND DISCUSSION}

At E13.5, Fgfr2 ${ }^{\text {UB-l- }}$ kidneys had more severe defects than Frs $2 \alpha^{U B-I-}$, but Fgfr2 ${ }^{U B-I-}$ and Fgfr2IFrs $2 \alpha^{U B-I-}$ kidneys appear similar

Although $F r s 2 \alpha^{U B-/}$ mice in this study were also heterozygous for $F g f r 2$, we observed no obvious differences between these embryonic kidneys compared with previously published $F r s 2 \alpha^{U B-/}$ embryos that were wild type at the Fgfr 2 locus (Sims-Lucas et al., 2009b). Likewise, we observed no differences in $F g \mathrm{Fr}^{\mathrm{UB-} / \mathrm{C}}$ embryonic kidneys in this study (that were heterozygous for $F r s 2 \alpha$ ) and kidneys from previously published $F g f r 2^{U B-\digamma}$ embryos (that were wild type for Frs2 $\alpha$ ) (Sims-Lucas et al., 2009a; Zhao et al., 2004).

Compared with controls, H\&E sections of E13.5 $\mathrm{Fg} f \mathrm{r} 2 / \mathrm{Frs} 2 \mathrm{\alpha}^{U B-/-}$ kidneys revealed dramatic decreases in ureteric branching, with large cortical regions devoid of ureteric tips, and with fewer developing nephrons (Fig. 1). Foxdl in situ hybridization revealed abnormal cortical stromal thickening in Fgfr $2 / F r s 2 \alpha^{U B-/}$ kidneys compared with controls (Fig. 1). Thus, at E13.5, Fgfr2/Frs $2 \alpha^{U B-/}$ mice had aberrant UB and cortical stromal development, appearing similar to $F g f r 2^{U B-/}$ kidneys, but more severely affected than $F r s 2 \alpha^{U B-/}$ kidneys (Sims-Lucas et al., 2009b; Zhao et al., 2004).
To quantitate differences among mutants, we generated 3D reconstructions of E13.5 kidneys and ureteric trees. Mean kidney surface area, length and volume of $F g f r 2 / F r s 2 \alpha^{U B-/}$ and

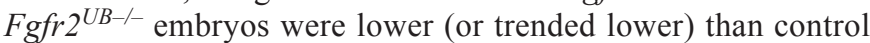
and $F r s 2 \alpha^{U B-/}$ embryos (Fig. 2; see Fig. S1 and Table S3 in the supplementary material). Fgfr $2 / F r s 2 \alpha^{U B-/}$ and $F g f r 2^{U B-/-}$ kidney sizes were not statistically different, however, as was the case between control and Frs $2 \alpha^{U B-/}$ kidneys. 3D reconstructed ureteric trees showed that, compared with controls, Frs $2 \alpha^{U B-I}$ kidneys had mild branching defects (fewer tips and branches and less ureteric tissue per kidney volume), whereas $F g f r 2 / F r s 2 \alpha^{U B-/}$ and $F g f r 2^{U B-/-}$ had more severe defects (Fig. 2; see Movies 1-4 in the supplementary material). Compared with controls, mean ureteric volume and surface areas (absolute and relative) were modestly reduced in $\operatorname{Frs} 2 \alpha^{U B-/-}$ kidneys and

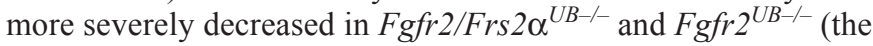
latter two were not statistically different from each other) (Fig. 2; see Fig. S1 and Table S3 in the supplementary material). Images of skeletonized ureteric trees reinforced observations that, compared with controls, Frs $2 \alpha^{U B-/-}$ kidneys had moderate branching defects, but $\mathrm{Fg} f \mathrm{r} 2 / \mathrm{Frs} 2 \mathrm{\alpha}^{\mathrm{UB-/}}$ and $\mathrm{Fg} f \mathrm{r}^{\mathrm{UB-/}}$ had even more severe defects (Fig. 2; see Movies 5-8 in the supplementary material). Measurements of skeletonized trees confirmed that
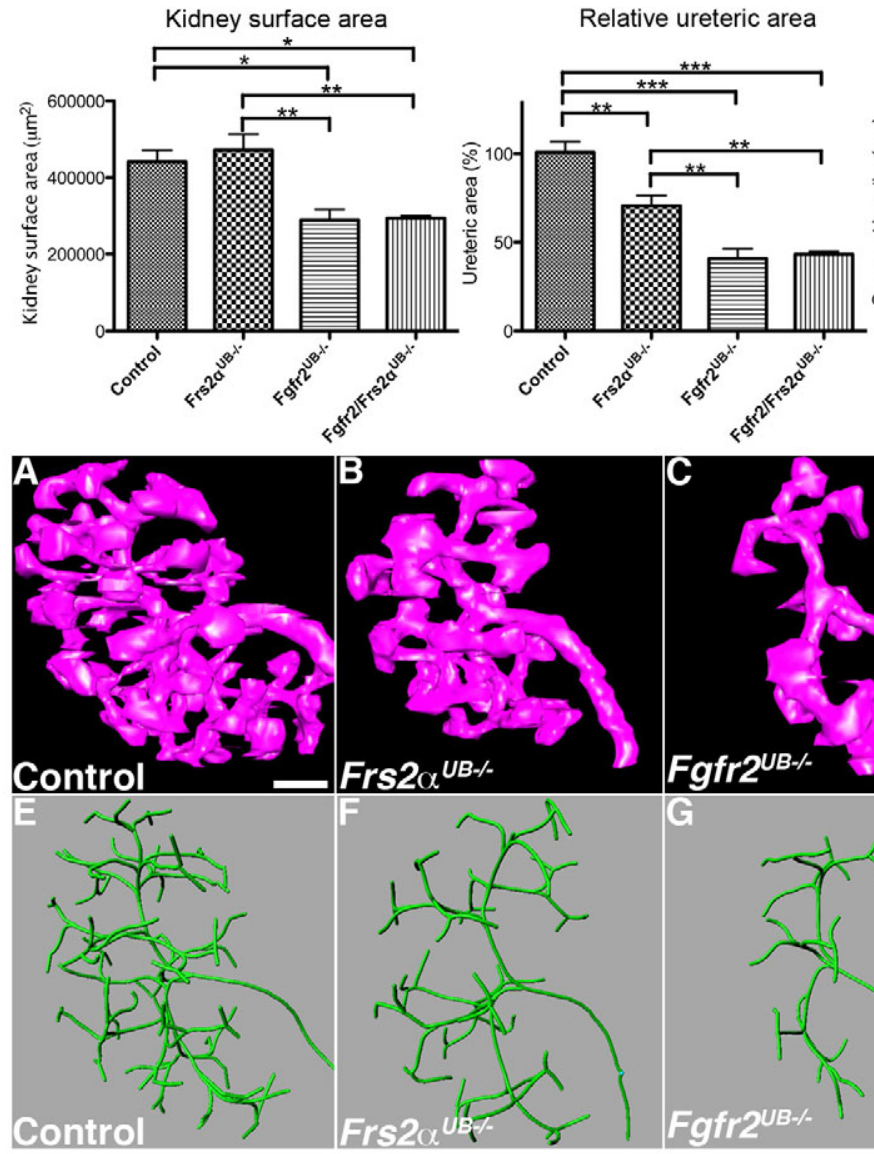
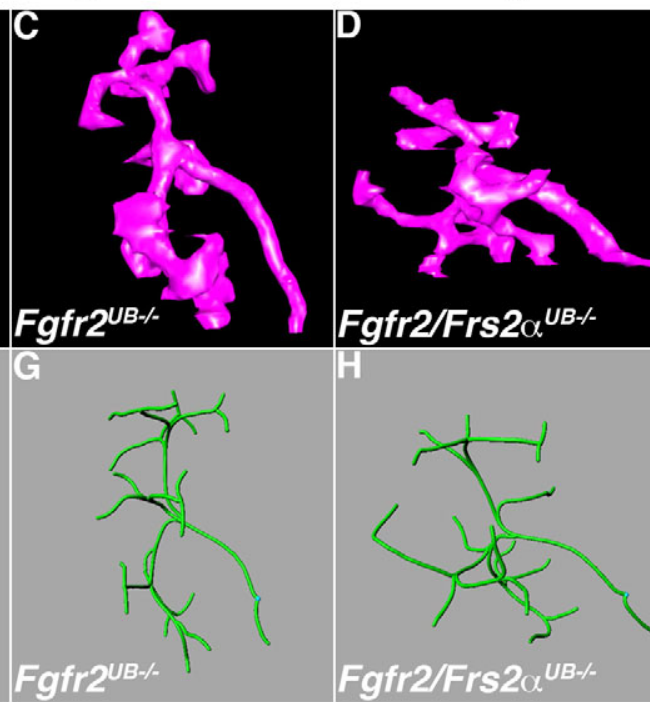

Fig. 2. 3D reconstructions in E13.5 Fgfr2/Frs2 $\alpha^{\text {UB-I- }}$ Fgfr2 UB-I- and Frs $2 \alpha^{\text {UB-I- }}$ kidneys. Top left: Graph demonstrates that control ( $\left.n=4\right)$ and Frs $2 \alpha^{U B-/-}(n=5)$ kidneys are larger than Fgfr2 ${ }^{U B--}(n=4)$ and Fgfr2/Frs $2 \alpha^{U B-/-}(n=3)$ kidneys and that the latter two are not statistically different from each other. Top middle and right: Graphs demonstrate that relative ureteric surface area and ureteric segment lengths are largest in controls followed by Frs $2 \alpha^{U B-/-}$, and then by Fgfr2 ${ }^{U B-/-}$ and Fgfr2/Frs $2 \alpha^{U B-/-}$. ${ }^{*} P<0.05 ;{ }^{*} P<0.01 ; * * * P<0.001$. (A-H) Images of 3D reconstructions show that, compared with controls $(A, E)(n=4)$, Frs $2 \alpha^{U B-l-}(B, F)(n=5)$ have moderate ureteric branching defects, including fewer tips and branches as well as proportionally less ureteric tissue per kidney volume. The branching defects are more severe in Fgfr2 ${ }^{\cup B-l-}(C, G)(n=4)$ and Fgfr2/Frs2 $\alpha^{U B-I-}(D, H)$ $(n=3)$ mice. Scale bar: $100 \mu \mathrm{m}$. 


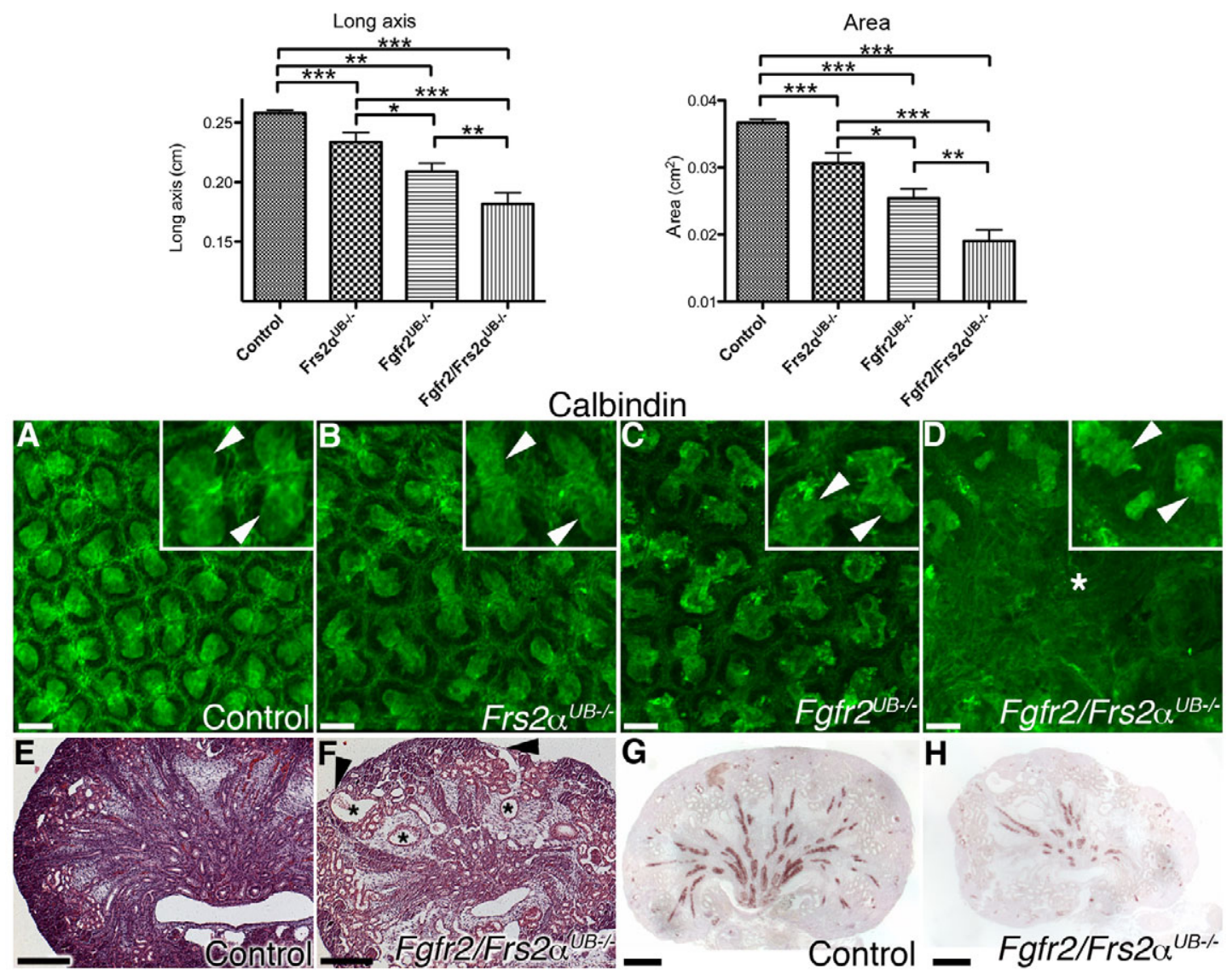

Fig. 3. E17.5 Fgfr2/Frs $2 \alpha^{\text {UB-I- }}$, Fgfr2 ${ }^{\text {UB-I- }}$ and Frs2 $\alpha^{\text {UB-I- }}$ kidneys. Top: Graphs demonstrate decreasing kidney long axis and cross-sectional area (area) from control to Frs $2 \alpha^{U B-1-}$ to Fgfr2 ${ }^{U B-1-}$ to Fgfr2/Frs $2 \alpha^{U B-1-}$ mice. ${ }^{*} P<0.05 ;{ }^{* *} P<0.01 ;{ }^{* * *} P<0.001$. (A-D) Calbindin whole-mount immunofluorescence demonstrates worsening UB defects from control (A) to Frs $2 \alpha^{U B-/-}$ (B) to Fgfr2 ${ }^{U B-/-}$ (C) to Fgfr2/Frs $2 \alpha^{U B-/-}$ mice (D), including wider spaces between tips, distorted tip shapes (arrowheads in upper right corners show $2 \times$ magnified tips), and large areas devoid of tips in Fgfr2/Frs $2 \alpha^{U B-1-}$ kidneys (asterisk). (E-H) H\&E stains show that, compared with controls (E), Fgfr2/Frs2 $\alpha^{U B-l-}$ (F) have distorted renal architecture including thin renal cortex (arrowheads) overlying dilated tubules (asterisks). Aquaporin 2 immunostaining demonstrates that, compared with controls (G), Fgfr2/Frs $2 \alpha^{U B-1-}$ mutants $(H)$ have fewer collecting ducts. Scale bars: $100 \mu \mathrm{m}$ for A-D; $250 \mu \mathrm{m}$ for E-H.

mean total ureteric segment lengths and numbers (branches, tips and all segments) were greatest in controls, followed by Frs $2 \alpha^{U B-/-}$, and then Fgfr $2 / F r s 2 \alpha^{U B-/-}$ and $F g f r 2^{U B-/-}$ (the latter two were not statistically different from each other) (Fig. 2 and see Table S3 in the supplementary material). Thus, 3D reconstructions revealed that, at E13.5, ureteric branching defects in $F r s 2 \alpha^{U B-/-}$ kidneys were milder than in $F g f r 2^{U B-/-}$; however, there were no detectable differences between Fgfr $2 / F r s 2 \alpha^{U B-/-}$ and Fgfr $2^{U B-/-}$. These findings could be consistent with Frs $2 \alpha$ signaling downstream of Fgfr2, but clearly suggest that Fgfr2 signals through molecules other than Frs $2 \alpha$ in the UB, including candidates such as phospholipase $\mathrm{C} \gamma$ (PLC $\gamma$; Plcg1 - Mouse Genome Informatics), Crk2 (Cdk6 Mouse Genome Informatics) and Src homology 2 domaincontaining adapter protein B (Shb) (Eswarakumar et al., 2005; Lundin et al., 2003; Powers et al., 2000). Although all of the latter three molecules are expressed in kidney, PLC $\gamma$ is a strong candidate for transmitting Fgfr2 signaling in the UB because mice chimeric for $P L C \gamma 1^{-/-}$and wild-type cells develop severe renal dysplasia (Prosser et al., 2003; Shirane et al., 2001; Welsh et al., 1994).

\section{At later stages, Fgfr2/Frs2 $\alpha^{\text {UB-I- }}$ kidneys are more severely affected than either Fgfr $2^{U B-I-}$ or Frs $2 \alpha^{\text {UB-I- }}$ kidneys}

At E17.5, although embryo sizes were comparable among all genotypes (not shown), both Frs $2 \alpha^{U B-/}$ and $F g f r 2^{U B-/-}$ had smaller kidneys than controls by long axis and cross-sectional area (Fig. 3; see Table S4 in the supplementary material). However, Fgfr $2 / F r s 2 \alpha^{U B-/}$ mutants had dramatically smaller kidneys than any other genotype (including $F g f r 2^{U B-/-}$ ). Whole-mount calbindin immunostaining demonstrated that, compared with controls, $F r s 2 \alpha^{U B-/}$ mice had relatively normal ureteric tip shapes, although there were slightly larger distances between tips (Fig. 3). $F g f r 2^{U B-/}$ kidneys had ureteric tips with aberrant shapes and longer distances between tips than kidneys of $F r s 2 \alpha^{U B-/-}$ mice (Fig. 3). Fgfr $2 / F r s 2 \alpha^{U B-/-}$ mice, however, had more severe defects than all other genotypes with large regions devoid of tips (Fig. 3). E17.5 histological sections revealed that $F g f r 2 / F r s 2 \alpha^{U B-/-}$ kidneys were disorganized with regions of outer cortex that were thin near areas of tubular dilatation (Fig. 3). Immunostaining for aquaporin 2 also showed that compound mutants had fewer collecting ducts than controls (Fig. 3). 


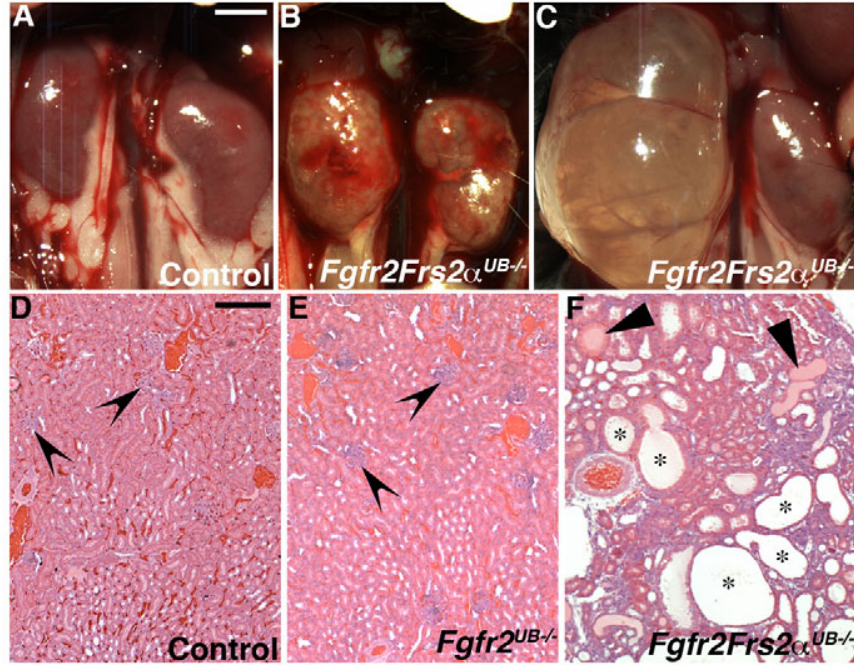

Fig. 4. P30 control and Fgfr2/Frs $2 \alpha^{\mathrm{UB}-/-}$ kidneys in situ hybridization and histological sections. (A-C) Compared with controls (A), Fgfr2/Frs $2 \alpha^{U B-1-}$ mutants often have a translucent or cystic appearance (B) or frank hydronephrosis (C). (D-F) Compared with controls (D), Fgfr2 ${ }^{U B-1-}$ kidneys (E) appear similar except that the glomeruli (concave arrowheads) are larger. By contrast, Fgfr2/Frs $2 \alpha^{U B-1-}$ kidneys (F) are dysplastic with many cysts (asterisks) and have tubules filled with proteinaceous material (arrowheads). Scale bar: $0.25 \mathrm{~cm}$ for A-C; $150 \mu \mathrm{m}$ for D-F.

By post-natal day 30 (P30), Fgfr2/Frs $2 \alpha^{U B-/-}$ kidneys were significantly abnormal appearing translucent/cystic or frankly hydronephrotic (Fig. 4). Histological sections revealed that mutant kidneys had cystic dysplasia with tubular atrophy and proteinaceous casts (Fig. 4). Although some Fgfr ${ }^{U B-/-}$ kidneys developed hydronephrosis (not shown), the parenchyma of these mutants had a relatively normal appearance (Fig. 4). Whereas Frs $2 \alpha^{U B-/-}$ and $F g f r 2^{U B-/-}$ glomeruli were larger than controls (by cross-sectional area and perimeter), Fgfr $2 / F r s 2 \alpha^{U B-/-}$ glomeruli were larger than all other genotypes (see Fig. S2 in the supplementary material). Thus, beyond E13.5, Fgfr $2 / F r s 2 \alpha^{U B-/}$ had more severe kidney defects than all other genotypes. These findings strongly suggest additive roles for Frs $2 \alpha$ and $F g f r 2$ in the UB. The divergence in Fgfr $2^{U B-/-}$ and Fgfr $2 / F r s 2 \alpha^{U B-/-}$ renal abnormalities at later stages could still be consistent with Frs $2 \alpha$ transmitting Fgfr2 signaling; however, it is possible that at early stages of ureteric and kidney morphogenesis, our assays were not sensitive enough to detect differences and that the two molecules signal independently.

\section{Fgfr $1^{\text {UB-I-Fgfr2 }}{ }^{\text {LRILR }}$ kidneys are phenotypically normal}

Fgfr $2^{L R / L R}$ mice with point mutations in the Frs $2 \alpha$ binding site on Fgfr2 had normal kidneys, suggesting independent roles of Fgfr2 and Frs $2 \alpha$ in the UB (Sims-Lucas et al., 2009b). Although Fgfr1 is present at low levels in the ureteric epithelium (as opposed to Fgfr2), wild-type Fgfr1 could form heterodimers with mutant Fgfr2, thus recruiting Frs $2 \alpha$ (Poladia et al., 2006; Zhao et al., 2004). To avoid potential rescue by Fgfrl, mice were generated with conditional UB deletion of $F g f r l$ and with $F g f r 2^{L R / L R}$ point mutations. E15.5 Fgfr $1^{U B-/} F g f r 2^{L R / L R}$ mice had normal ureteric branching by whole mount Ret in situ hybridization (see Fig. S3 in the supplementary material). E17.5 Fgfr $1^{U B-/} F g f r 2^{L R / L R}$ kidneys had normal histology and size (see Fig. S3 and Table S5 in the supplementary material). Thus, blockade of Fgfr2 signaling through Frs $2 \alpha$ in the UB does not affect kidney development, even with loss of Fgfr1. Wild-type Fgfr3 and/or Fgfr4 could also hypothetically form heterodimers with $\mathrm{Fgfr} 2^{\mathrm{LR}}$ and rescue kidney abnormalities; however, these receptors are also expressed at low levels in UB like Fgfrl (www.eurexpress.org/ee/). Thus, the most likely explanation is that Fgfr2 and Frs $2 \alpha$ act independently in the UB.

Despite the presence of normal kidneys in $F g f r 1^{U B--}-F g f r 2^{L R / L R}$, $\operatorname{Frs} 2 \alpha^{U B-/-}$ mice have aberrant ureteric branching; thus, other RTKs must utilize Frs $2 \alpha$ in the UB. Candidate RTKs with docking sites for Frs $2 \alpha$ are Ret, neurotrophin receptors (Trks) and anaplastic lymphoma kinase (Alk). Ret is co-expressed with Frs $2 \alpha$ in ureteric tips from E12.5 onward (unlike Fgfr2, which is expressed throughout the UB) (Hains et al., 2008; Pachnis et al., 1993; SimsLucas et al., 2009b; Zhao et al., 2004); furthermore, mice with point mutations in the Ret docking site for Frs $2 \alpha$ (and other adapter proteins) have renal hypoplasia, similar to $F r s 2 \alpha^{U B-/-}$ mice (Jijiwa et al., 2004). Among Trks (Alpers et al., 1993; Durbeej et al., 1993; Wheeler et al., 1998), only $\operatorname{Trk} B^{-/}$mice have renal abnormalities in the juxtaglomerular apparatus (Garcia-Suarez et al., 2006). Kidneys of $A l k^{-/}$mice have a normal appearance.

\section{Conclusion}

Although Fgfr 2 and Frs $2 \alpha$ have crucial roles in regulating UB morphogenesis, they appear to act in an independent and additive manner.

\section{Acknowledgements}

The authors thank Dr David Ornitz for the use of the floxed Fgfr2 mouse line. This study was supported by the National Institute of Diabetes and Digestive and Kidney Diseases Grant R01-DK070030 (C.M.B.) and National Institutes of Health program Grant P50 AR054086 (V.P.E.). Deposited in PMC for release after 12 months.

\section{Competing interests statement}

The authors declare no competing financial interests.

\section{Supplementary material}

Supplementary material for this article is available at

http://dev.biologists.org/lookup/suppl/doi:10.1242/dev.062158/-/DC1

\section{References}

Alpers, C. E., Hudkins, K. L., Ferguson, M., Johnson, R. J., Schatteman, G. C. and Bothwell, M. (1993). Nerve growth factor receptor expression in fetal, mature, and diseased human kidneys. Lab. Invest. 69, 703-713.

Barasch, J., Qiao, J., McWilliams, G., Chen, D., Oliver, J. A. and Herzlinger, D. (1997). Ureteric bud cells secrete multiple factors, including bFGF, which rescue renal progenitors from apoptosis. Am. J. Physiol. 273, F757-F767.

Barasch, J., Yang, J., Ware, C. B., Taga, T., Yoshida, K., Erdjument-Bromage, H., Tempst, P., Parravicini, E., Malach, S., Aranoff, T. et al. (1999). Mesenchymal to epithelial conversion in rat metanephros is induced by LIF. Cell 99, 377-386.

Celli, G., LaRochelle, W. J., Mackem, S., Sharp, R. and Merlino, G. (1998). Soluble dominant-negative receptor uncovers essential roles for fibroblast growth factors in multi-organ induction and patterning. EMBO J. 17, 16421655

Costantini, F. and Kopan, R. (2010). Patterning a complex organ: branching morphogenesis and nephron segmentation in kidney development. Dev. Cell 18, 698-712.

Durbeej, M., Soderstrom, S., Ebendal, T., Birchmeier, C. and Ekblom, P. (1993). Differential expression of neurotrophin receptors during renal development. Development 119, 977-989.

Eswarakumar, V. P., Lax, I. and Schlessinger, J. (2005). Cellular signaling by fibroblast growth factor receptors. Cytokine Growth Factor Rev. 16, 139-149.

Eswarakumar, V. P., Ozcan, F., Lew, E. D., Bae, J. H., Tome, F., Booth, C. J., Adams, D. J., Lax, I. and Schlessinger, J. (2006). Attenuation of signaling pathways stimulated by pathologically activated FGF-receptor 2 mutants prevents craniosynostosis. Proc. Natl. Acad. Sci. USA 103, 18603-18608. 
Garcia-Suarez, O., Gonzalez-Martinez, T., Germana, A., Monjil, D. F., Torrecilla, J. R., Laura, R., Silos-Santiago, I., Guate, J. L. and Vega, J. A. (2006). Expression of TrkB in the murine kidney. Microsc. Res. Tech. 69, 1014-1020.

Gotoh, N. (2008). Regulation of growth factor signaling by FRS2 family docking/scaffold adaptor proteins. Cancer Sci. 99, 1319-1325.

Hadari, Y. R., Gotoh, N., Kouhara, H., Lax, I. and Schlessinger, J. (2001). Critical role for the docking-protein FRS2 alpha in FGF receptor-mediated signal transduction pathways. Proc. Natl. Acad. Sci. USA 98, 8578-8583.

Hains, D., Sims-Lucas, S., Kish, K., Saha, M., McHugh, K. and Bates, C. M. (2008). Role of fibroblast growth factor receptor 2 in kidney mesenchyme. Pediatr. Res. 64, 592-598.

Jijiwa, M., Fukuda, T., Kawai, K., Nakamura, A., Kurokawa, K., Murakumo, Y., Ichihara, M. and Takahashi, M. (2004). A targeting mutation of tyrosine 1062 in Ret causes a marked decrease of enteric neurons and renal hypoplasia. Mol. Cell. Biol. 24, 8026-8036.

Li, Z., Jerebtsova, M., Liu, X. H., Tang, P. and Ray, P. E. (2006). Novel cystogenic role of basic fibroblast growth factor in developing rodent kidneys. Am. J. Physiol. Renal Physiol. 291, 289-296.

Lin, Y., Zhang, J., Zhang, Y. and Wang, F. (2007). Generation of an Frs2alpha conditional null allele. Genesis 45, 554-559.

Lundin, L., Ronnstrand, L., Cross, M., Hellberg, C., Lindahl, U. and ClaessonWelsh, L. (2003). Differential tyrosine phosphorylation of fibroblast growth factor (FGF) receptor-1 and receptor proximal signal transduction in response to FGF-2 and heparin. Exp. Cell Res. 287, 190-198.

Nguyen, H. Q., Danilenko, D. M., Bucay, N., DeRose, M. L., Van, G. Y., Thomason, A. and Simonet, W. S. (1996). Expression of keratinocyte growth factor in embryonic liver of transgenic mice causes changes in epithelial growth and differentiation resulting in polycystic kidneys and other organ malformations. Oncogene 12, 2109-2119.

Ohuchi, H., Hori, Y., Yamasaki, M., Harada, H., Sekine, K., Kato, S. and Itoh N. (2000). FGF10 acts as a major ligand for FGF receptor 2 Illb in mouse multiorgan development. Biochem. Biophys. Res. Commun. 277, 643-649.

Pachnis, V., Mankoo, B. and Costantini, F. (1993). Expression of the c-ret protooncogene during mouse embryogenesis. Development 119, 1005-1017.

Perantoni, A. O., Dove, L. F. and Karavanova, I. (1995). Basic fibroblast growth factor can mediate the early inductive events in renal development. Proc. Natl. Acad. Sci. USA 92, 4696-4700

Poladia, D. P., Kish, K., Kutay, B., Hains, D., Kegg, H., Zhao, H. and Bates, C. M. (2006). Role of fibroblast growth factor receptors 1 and 2 in the metanephric mesenchyme. Dev. Biol. 291, 325-339.

Powers, C. J., McLeskey, S. W. and Wellstein, A. (2000). Fibroblast growth factors, their receptors and signaling. Endocr. Relat. Cancer 7, 165-197.
Prosser, S., Sorokina, E., Pratt, P. and Sorokin, A. (2003). Crklll: a novel and biologically distinct member of the Crk family of adaptor proteins. Oncogene 22, 4799-4806.

Qiao, J., Uzzo, R., Obara-Ishihara, T., Degenstein, L., Fuchs, E. and Herzlinger, D. (1999). FGF-7 modulates ureteric bud growth and nephron number in the developing kidney. Development 126, 547-554

Qiao, J., Bush, K. T., Steer, D. L., Stuart, R. O., Sakurai, H., Wachsman, W. and Nigam, S. K. (2001). Multiple fibroblast growth factors support growth of the ureteric bud but have different effects on branching morphogenesis. Mech Dev. 109, 123-135.

Revest, J. M., Spencer-Dene, B., Kerr, K., De Moerlooze, L., Rosewell, I. and Dickson, C. (2001). Fibroblast growth factor receptor 2-Illb acts upstream of Shh and Fgf4 and is required for limb bud maintenance but not for the induction of Fgf8, Fgf10, Msx1, or Bmp4. Dev. Biol. 231, 47-62.

Schuchardt, A., D'Agati, V., Larsson-Blomberg, L., Costantini, F. and Pachnis, V. (1994). Defects in the kidney and enteric nervous system of mice lacking the tyrosine kinase receptor Ret [see comments]. Nature 367, 380-383.

Shirane, M., Sawa, H., Kobayashi, Y., Nakano, T., Kitajima, K., Shinkai, Y. Nagashima, K. and Negishi, I. (2001). Deficiency of phospholipase C-gamma1 impairs renal development and hematopoiesis. Development 128, 5173-5180.

Sims-Lucas, S., Argyropoulos, C., Kish, K., McHugh, K., Bertram, J. F. Quigley, R. and Bates, C. M. (2009a). Three-dimensional imaging reveals ureteric and mesenchymal defects in Fgfr2-mutant kidneys. J. Am. Soc. Nephrol. 20, 2525-2533

Sims-Lucas, S., Cullen-McEwen, L., Eswarakumar, V. P., Hains, D., Kish, K., Becknell, B., Zhang, J., Bertram, J. F., Wang, F. and Bates, C. M. (2009b) Deletion of Frs2alpha from the ureteric epithelium causes renal hypoplasia. Am J. Physiol. Renal Physiol. 297, F1208-F1219.

Welsh, M., Mares, J., Karlsson, T., Lavergne, C., Breant, B. and ClaessonWelsh, L. (1994). Shb is a ubiquitously expressed Src homology 2 protein. Oncogene 9, 19-27.

Wheeler, E., Gong, H., Grimes, R., Benoit, D. and Vazquez, L. (1998). p75NTR and Trk receptors are expressed in reciprocal patterns in a wide variety of nonneural tissues during rat embryonic development, indicating independent receptor functions. J. Comp. Neurol. 391, 407-428.

Yu, K., Xu, J., Liu, Z., Sosic, D., Shao, J., Olson, E. N., Towler, D. A. and Ornitz, D. M. (2003). Conditional inactivation of FGF receptor 2 reveals an essential role for FGF signaling in the regulation of osteoblast function and bone growth. Development 130, 3063-3074.

Zhao, H., Kegg, H., Grady, S., Truong, H. T., Robinson, M. L., Baum, M. and Bates, C. M. (2004). Role of fibroblast growth factor receptors 1 and 2 in the ureteric bud. Dev. Biol. 276, 403-415. 